\title{
The Crosstalk between IncRNA-SNHG7/miRNA- 181/cbx7 Modulates Malignant Character in Lung Adenocarcinoma
}

\author{
Yao-fei Pei, ${ }^{*}$ Yi He, ${ }^{*}$ Li-zhen Hu, ${ }^{\dagger}$ Bing Zhou, ${ }^{\ddagger}$ He-yun $\mathrm{Xu}^{\ddagger}{ }^{\ddagger}$ and Xi-qiang Liu ${ }^{\S థ \|}$
}

\begin{abstract}
From the Department of Thoracic Surgery, ${ }^{*}$ Shanghai Chest Hospital, Shanghai Jiaotong University, Shanghai; the Outpatient Department, ${ }^{\dagger}$ Hunan Provincial People’s Hospital, The First Affiliated Hospital of Hunan Normal University, Changsha; the Departments of Cardiothoracic Surgery and Hepatopancreatobiliary Surgery and Minimally Invasive Surgery, ${ }^{\S}$ Zhejiang Provincial People's Hospital, People's Hospital of Hangzhou Medical College, Hangzhou; the Key Laboratory of Tumor Molecular Diagnosis and Individualized Medicine of Zhejiang Province, "Hangzhou; and the Key Laboratory of Gastroenterology of Zhejiang Province, ${ }^{\|}$Hangzhou, P. R. China
\end{abstract}

\author{
Accepted for publication \\ February 18, 2020. \\ Address correspondence to Xi-qiang \\ Liu, M.D., Ph.D., Department of \\ Hepatobiliary-Pancreatic Surgery, or \\ He-yun Xu, M.D., Ph.D., Department \\ of Cardiothoracic Surgery, Zhejiang \\ Provincial People's Hospital (ZJPPH), \\ 14th Floor, Bldg. 1, 158 Shangtang \\ Rd., Hangzhou, Zhejiang Province \\ 310014, P. R. China. E-mail: \\ 416095688@qq.com or doctorpxhy@ \\ sina.com.
}

\begin{abstract}
Lung adenocarcinoma (LUAD) is a malignant tumor with poor patient survival and high patient mortality. Long noncoding RNA is profoundly involved in the tumorigenesis of LUAD. The present study explores the effect of small nucleolar RNA host gene 7 (SNHG7) on the progression of LUAD and its underlying mechanisms. SNHG7 was found to be down-regulated in LUAD tissues compared with normal tissues. Altered SNHG7 expression induced changes in cell proliferation and migration both in vitro and in vivo. Mechanistically, it was found that SNHG7 interacted with microRNA mir-181 and sequentially up-regulated $c b \times 7 . c b \times 7$, which suppresses the Wnt/ $\beta$-catenin pathway in LUAD, was found to be a direct target of mir-181. Taken together, loss of SNHG7 in LUAD up-regulated mir-181 and then down-regulated the tumor suppressor cbx7. (Am J Pathol 2020, 190: 1343-1354; https://doi.org/10.1016/ j.ajpath.2020.02.011)
\end{abstract}

Lung adenocarcinoma (LUAD) is one of the most lethal malignant tumors worldwide. ${ }^{1-3}$ Despite developments in surgical and chemotherapeutic treatments, the prognosis of patients with LUAD is still poor due to metastasis and recurrence. However, the mechanisms that induce metastasis are still unclear. Therefore, it is necessary to investigate new therapeutic targets or prognostic markers related to the tumorigenesis and metastasis of LUAD.

Long noncoding RNAs (lncRNAs) are non-proteincoding RNAs, which are longer than 200 nucleic acids. Accumulating evidence has indicated the involvement of IncRNA in tumor proliferation and metastasis. Aberrantly expressed lncRNAs were observed in different types of cancers. For example, HOTAIR is seen in hepatocellular carcinoma, PVT1 in colorectal cancer, and H19 in gastric cancer. ${ }^{4-6}$ IncRNA plays its critical role through various mechanisms such as lncRNA-miRNA interaction, lncRNA - protein interaction, and epigenetic modulation. In lncRNA-miRNA interaction, lncRNA acts as a competing endogenous RNA (ceRNA) and sponges miRNA. ${ }^{7}$ In other words, lncRNA and mRNA affect each other through competitively combining with miRNA response elements (MREs). In this process, IncRNA regulates the level of target mRNA through sequestering miRNA. Zhuang et $\mathrm{al}^{8}$ reported that the IncRNA XIST inhibited hepatocellular carcinoma by sequestering mir-92. The interaction between XIST and mir-92 increased smad7, a target of mir-92. HOTAIR promoted renal cell carcinoma progression via regulating mir-138-dependent $\mathrm{EZH} 2{ }^{9}$

The lncRNA small nucleolar RNA host gene 7 (SNHG7) has been reported as an oncogenic lncRNA in multiple types of tumors such as hepatocellular carcinoma, breast cancer,

\footnotetext{
Supported by the Zhejiang Lung Cancer Center, Natural Science Foundation of Zhejiang Province grant LY18H160038, and Medical Research Plan of Zhejiang Province grant 2016KYA032

Y.P. and Y.H. contributed equally to this work.

Disclosures: None declared.
} 
and colorectal cancer. ${ }^{10}$ SNHG7 was also found to promote malignant transformation in LUAD. ${ }^{11}$ However, in this current study, the authors found an anti-tumor effect of SNHG7 in LUAD. In this study, SNHG7 was found to be down-regulated in LUAD tissues as compared with nontumor tissues. Patients with high SNHG7 expression had a better prognosis. SNHG7 inhibited cell proliferation and migration in LUAD cells in vitro and in vivo. Further investigation revealed SNHG7 plays a role as an endogenous sponge of mir-181. mir-181 promotes LUAD cell proliferation and migration by targeting cbx7. SNHG7 abrogates the role of mir-181 in promoting LUAD proliferation and metastasis. Hence, the role of the SNHG7/mir-181/ cbx7 pathway in LUAD proliferation and migration was investigated.

\section{Materials and Methods}

\section{Tissues and Database}

The high-throughput data of LUAD patients were obtained from the Cancer Genome Atlas (TCGA) database (https:// portal.gdc.cancer.gov). A total of 112 pairs of tumor and adjacent nontumor tissues were used in this study. All samples were collected from LUAD patients who underwent surgery in the Zhejiang Provincial People's Hospital. Written informed consent was obtained from all patients. The present study was approved by the ethics committee of the Zhejiang Provincial People's Hospital.

\section{Cell Culture}

All human LUAD cell lines used in the present study were obtained from ATCC (Manassas, VA). These cell lines were cultured in RPMI 1640 medium containing $10 \%$ fetal bovine serum at $37^{\circ} \mathrm{C}$ with $5 \% \mathrm{CO}_{2}$.

\section{Colony Formation and Cell Migration}

Colony formation and cell migration assays were performed as previously described. ${ }^{12}$ All experiments were performed in triplicate.

\section{RNA Extraction and $q P C R$}

Total RNA was extracted using TRIzol reagent (Invitrogen, Carlsbad, CA). The RNA was then reverse transcribed to cDNA using a reverse transcription kit (Promega, Madison, WI). The quantitative PCR (qPCR) reaction was performed using the SYBR Green Mix (Applied Biosystems, Foster City, CA), and a miRNA cDNA Synthesis kit (TaKaRa Bio, Otsu, Japan) was used for the detection of miRNAs. GAPDH and U6 were used as endogenous controls for mRNA and miRNA detection, respectively. All primers used in the present study are listed in Table 1. All experiments were performed in triplicate.
Table 1 Primers for Real-Time PCR

\begin{tabular}{|c|c|}
\hline Construct & Sequence \\
\hline U6 & 5'-CTCGCTTCGGCAGCACA-3' \\
\hline \multirow[t]{2}{*}{ GAPDH } & F: 5'-CTGGGCTACACTGAGCACC-3' \\
\hline & R: 5'-AAGTGGTCGTTGAGGGCAATG-3' \\
\hline \multirow[t]{2}{*}{$C b \times 7$} & F: 5'-GCGTGCGGAAGGGTAAAGT-3' \\
\hline & R: 5'-GCTTGGGTTTCGGACCTCTC-3' \\
\hline \multirow[t]{2}{*}{ SNHG7 } & F: 5'-TTGCTGGCGTCTCGGTTAAT-3' \\
\hline & R: $5^{\prime}-$ GGAAGTCCATCACAGGCGAA-3' \\
\hline mir-181 & 5'-AACATTCAACGCTGTCGGTGAGT-3' \\
\hline \multirow[t]{4}{*}{ SNHG7 (BSP) } & F: 5'-GTTTTGATTTAGGT \\
\hline & AAGAGGTGAGG- $3^{\prime}$ \\
\hline & R: 5'-TCAAACCATAAACCAT \\
\hline & TСТСААТАС- $3^{\prime}$ \\
\hline SNHG7, primers & F: 5'-TTTATGAGGGAGGGG \\
\hline for DNA & TAGTAATATC-3' \\
\hline methylation & R: $5^{\prime}-\mathrm{AAAACGAAACCTAAA}$ \\
\hline detection & AATAAAACG- $3^{\prime}$ \\
\hline SNHG7 shRNA & 5'-GGTCAATCCTCCAATGTAA-3' \\
\hline$c b x 7$ shRNA & 5'-GGGTAAAGTCGAGTATCTG-3' \\
\hline Scramble shRNA & $5^{\prime}-$ TTCTCCGAACGTGTCACGT-3' \\
\hline
\end{tabular}

$F$, forward; $R$, reverse.

\section{Bisulfate Sequencing}

Genomic DNA was extracted and treated according to the guidelines of the Epi-kit (Qiagen, Hilden, Germany). The bisulfate-treated DNA was then amplified using PCR (Table 1). Afterward, the PCR products were subcloned into the vector. Subsequently, 10 clones of each group were randomly chosen and sent for sequencing. The sequencing results were analyzed using an online tool (Quantification Tool for Methylation Analysis, http://quma.cdb.riken.jp, last accessed September 9, 2019).

\section{Detection of DNA Methylation Content}

An EpiMark 5-mC analysis kit (New England Biolabs, Ipswich, MA) was used for the detection of 5-mC. Genomic DNA was incubated with T4 Phage b-glucosyltransferase, according to the manufacturer's instructions. The DNA was then digested with the HpaII and MspI enzymes. Afterward, the digested products were amplified by PCR. The primers used are listed in Table 1. The 5-mC content was calculated according to a previously described method. ${ }^{13}$

\section{Western Blots}

Western blots were performed as previously described. ${ }^{12}$ Antibodies used in our study are listed in Table 2.

\section{Co-Immunoprecipitation}

The cell line was cultured in 6-well plates. The cells were then harvested, and the co-immunoprecipitation was performed as previously described. ${ }^{12}$ 
Table 2 Antibodies for Western Blot

\begin{tabular}{|c|c|c|c|}
\hline Antibody & Concentration & Specificity & Company \\
\hline $\mathrm{Cb} \times 7$ & $1: 1000$ & Rabbit & $\begin{array}{l}\text { GeneTex } \\
\quad \text { (Irvine, CA) }\end{array}$ \\
\hline$\beta$-catenin & $1: 1000$ & Rabbit & CST (Danvers, MA) \\
\hline cyclin D1 & 1:1000 & Rabbit & CST \\
\hline c-myc & $1: 1000$ & Rabbit & CST \\
\hline TCF4 & $1: 1000$ & Rabbit & CST \\
\hline E-cadherin & $1: 1000$ & Rabbit & CST \\
\hline $\mathrm{N}$-cadherin & $1: 1000$ & Rabbit & CST \\
\hline MMP7 & $1: 1000$ & Rabbit & CST \\
\hline c-jun & 1:1000 & Rabbit & CST \\
\hline Vimentin & $1: 1000$ & Rabbit & CST \\
\hline ZEB1 & 1:1000 & Rabbit & GeneTex \\
\hline ZEB2 & $1: 1000$ & Rabbit & GeneTex \\
\hline fra-1 & $1: 1000$ & Rabbit & CST \\
\hline GAPDH & 1:1000 & Rabbit & CST \\
\hline GFP antibody & $20 \mu \mathrm{l}$ & Rabbit & GeneTex \\
\hline
\end{tabular}

CST, Cell Signaling Technology.

\section{Constructs}

The cbx7 and SNHG7 overexpression plasmids were purchased from Vigene Bioscience (Jinan, China) and GenePharma (Shanghai, China), respectively. The lentivirus used for mir-181 overexpression and knockdown was purchased from Genechem (Shanghai, China). The shRNA sequences (Table 1) were packed in lentiviruses that were used to stably knockdown SNHG7 and cbx7 in the LUAD cell lines.

\section{Luciferase Assay}

mir-181 mimics or mir-NC, pGL3 plasmids with wild-type or mutant cbx7 $3^{\prime}$ untranslated region ( $3^{\prime}$-UTR), and a Renilla vector were cotransfected into cells. After 48 hours, luciferase activity was determined using a dual-luciferase reporter assay system (Promega). Renilla luciferase activity was used as an endogenous control. All experiments were performed at least three times.

\section{In Situ Hybridization}

LUAD tissues and adjacent nontumor tissues were dewaxed. Tissues with prehybridization solution were then hybridized at $65^{\circ} \mathrm{C}$ for 1 hour with $500 \mathrm{ng} / \mathrm{mL} \mathrm{SNHG7} \mathrm{probe} \mathrm{hybrid-}$ ization solution (Dako, Carpinteria, CA). Tissues were then cultured in a wet box at $55^{\circ} \mathrm{C}$ for 2 hours, followed by washing. Next, tissues were put into nitro blue tetrazolium and 5-bromo-4-chloro-3-indolyl phosphate for 1 hour. Finally, double distilled water was used to terminate the reaction. The following steps were performed according to the Ribo lncRNA Probe Mix (RiboBio, Guangzhou, China).

\section{RIP}

The plasmids pcDNA-SNHG7-MS2, pcDNA-SNHG7-mutMS2, pcDNA-H19-MS2, and pcDNA-MS2 were constructed and then transfected into LUAD cells. Cells were collected for RNA immunoprecipitation (RIP) using green fluorescent protein (GFP) antibody and a RNA immunoprecipitation kit (EMD Millipore, Burlington, MA). mir-181 level was detected by qPCR.

For argonaute 2 (Ago2)-based RIP assay, cells were harvested and lysed using buffer in a RIP kit (EMD Millipore). The cell lysate was then incubated with magnetic beads coated with anti-Ago2 antibody (EMD Millipore). The lysate was treated with proteinase K. Finally, immunoprecipitated RNA was purified and detected by qPCR.

\section{RNA Pulldown}

Biotinylated miRNAs were transfected into LUAD cells. After 72 hours, cells were collected. Cell lysate was incubated with streptavidin-coated magnetic beads (Pierce Biotechnology, Rockford, IL). The enriched RNAs were then purified by TRIzol and subjected to qPCR.

The probe complementary to SNHG7 was biotin-labeled. LUAD cells were transfected with SNHG7, SNHG7-mut, and H19. After 72 hours, cell lysates were incubated with biotinlabeled probe and streptavidin magnetic beads. The enriched miRNAs were then purified by TRIzol and subjected to qPCR.

\section{Animal Model}

Six-week-old male BALB/c nude mice were purchased from the Shanghai SLAC Laboratory Animal Co., Ltd. (Shanghai, China) and kept in specific pathogen-free conditions. The SNHG7 knockdown and mir-181 overexpressing PC9 cell lines were subcutaneously injected into the mice at a concentration of $2 \times 10^{6} / 100 \mu \mathrm{L}$ per mouse. All mice were euthanized at the end of the experiment. The tumor grafts from the mice were fixed. Tumors were measured and recorded every 5 days, and the tumor volumes were calculated as previously described. $^{14}$ The animal experiments were performed according to the Guide for the Care and Use of Laboratory Animals of the Zhejiang Provincial People's Hospital.

\section{Statistical Analysis}

The statistical analysis was performed using SPSS software version 19.0 (IBM Corporation, Armonk, NY). The Pearson correlation coefficient and the $\chi^{2}$ test were used to analyze the relationships. Other experimental data were analyzed via $t$-test. Values were considered statistically significant when $P<0.05$.

\section{Results}

SNHG7 Is Down-Regulated in LUAD and Associated with Poor Survival

Integrated analysis of the TCGA and Genotype-Tissue Expression (GTEx) databases showed that lncRNA 


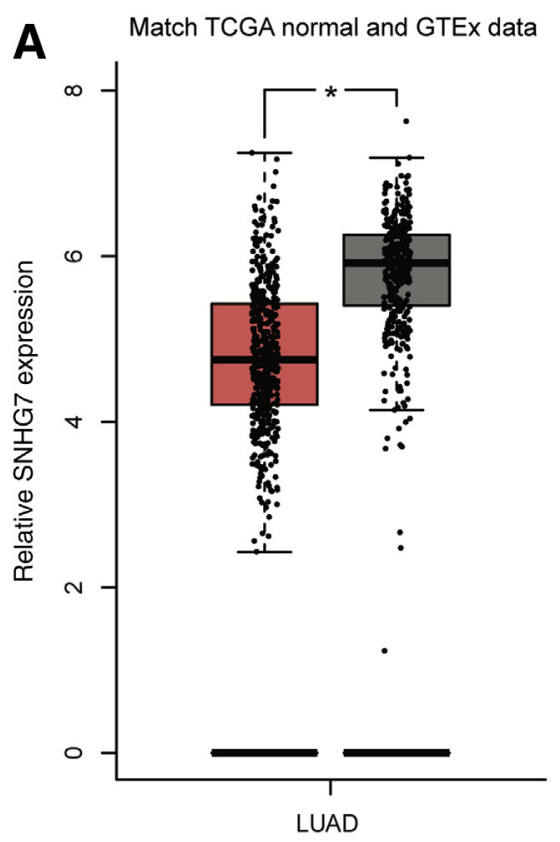

B

\section{C}
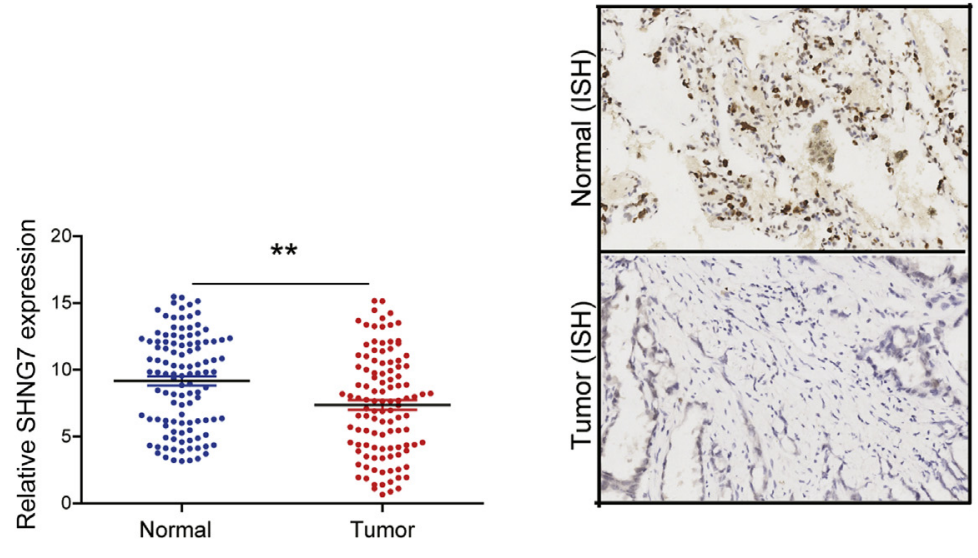

D TCGA
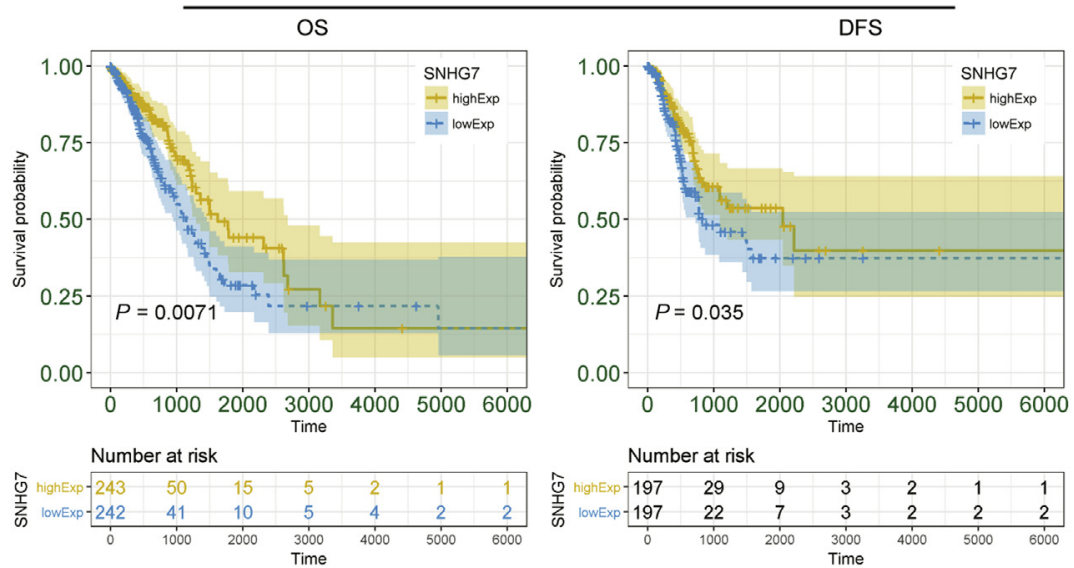

$\mathbf{E}$

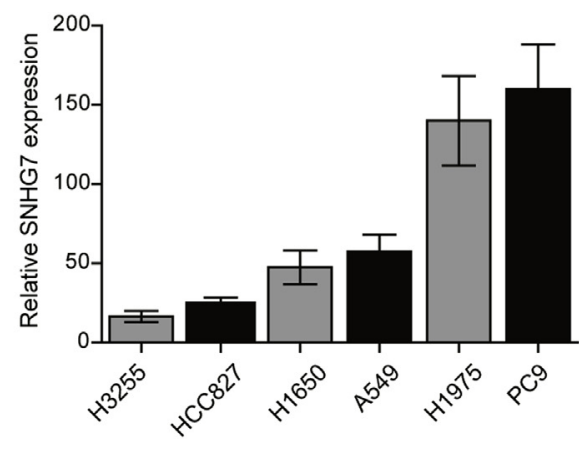

Figure 1 Small nucleolar RNA host gene 7 (SNHG7) is down-regulated in lung adenocarcinoma (LUAD) and associated with poor survival. A: SNHG7 is down-regulated in LUAD in the Cancer Genome Atlas (TCGA) and Genotype-Tissue Expression (GTEx) databases. Black lines in graphs stand for mean expression. B: SNHG7 expression in clinical specimen is shown. Black lines indicate the mean expression. C: In situ hybridization (ISH) of tumor and nontumor tissues. D: Kaplan-Meier analysis of LUAD patients with SNHG7 high expression (highExp) and low expression (lowExp). E: SNHG7 expression in cell lines. $n=$ 483 normal (A); $n=347$ tumor (A); $n=112$ normal and tumor (B). ${ }^{*} P<0.05,{ }^{*} P<0.01$. Original magnification, $\times 200$ (C). DFS, disease free survival; $\mathrm{N}$, normal; $0 \mathrm{~S}$, overall survival; $\mathrm{T}$, tumor.

SNHG7 is down-regulated in tumor tissues as compared with normal tissues (Figure $1 \mathrm{~A}$ and Supplemental Figure S1). To validate this result, SNHG7 expression was analyzed by qPCR in 112 pairs of tumor tissues and adjacent nontumor tissues that were obtained from LUAD patients. SNHG7 is significantly down-regulated in tumor tissues compared with nontumor tissues (Figure 1B). In situ hybridization also showed down-regulated SNHG7 in tumor tissues (Figure 1C). Kaplan-Meier analysis using data from the TCGA database showed that SNHG7 high expression indicated better prognosis in LUAD patients
(Figure 1D). Finally, SNHG7 expression was also detected in LUAD cell lines. H3255 has the lowest expression of SNHG7, whereas PC9 has the highest expression of SNHG7 (Figure 1E).

\section{SNHG7 Is a Direct Target of mir-181}

lncRNA always plays a role as a ceRNA by sponging miRNA. ${ }^{15}$ Through bioinformatics analysis, mir-181 was chosen as a target of lncRNA SNHG7 based on its involvement in carcinogenesis in LUAD. ${ }^{2}$ To validate this 
A

WT SNHG7: 5' CugCCCCGUGAACGUUUGAAUGUU 3' mir-181: 3' ugaGUGGCUGUCGC--AACUUACAL 5 Mut SNHG7: 5 ' cugCCGGCUGAAGCUAACUUACAu 3

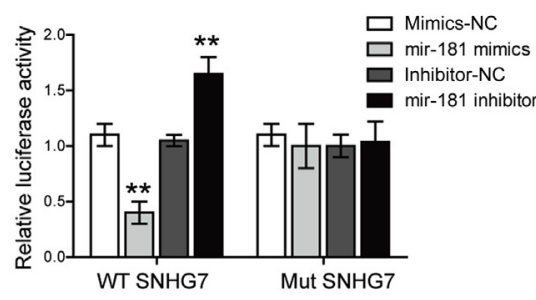

D
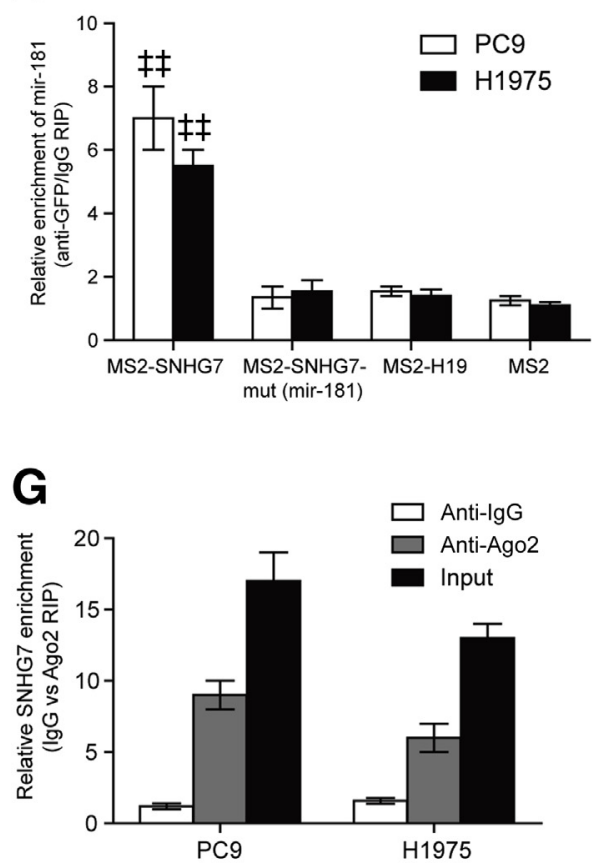

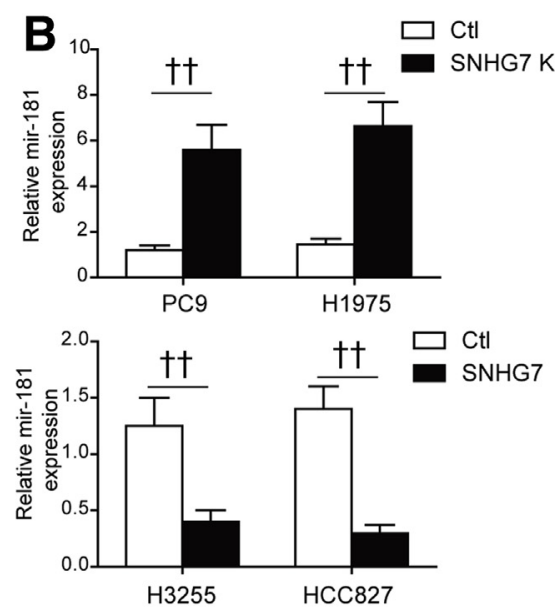

E
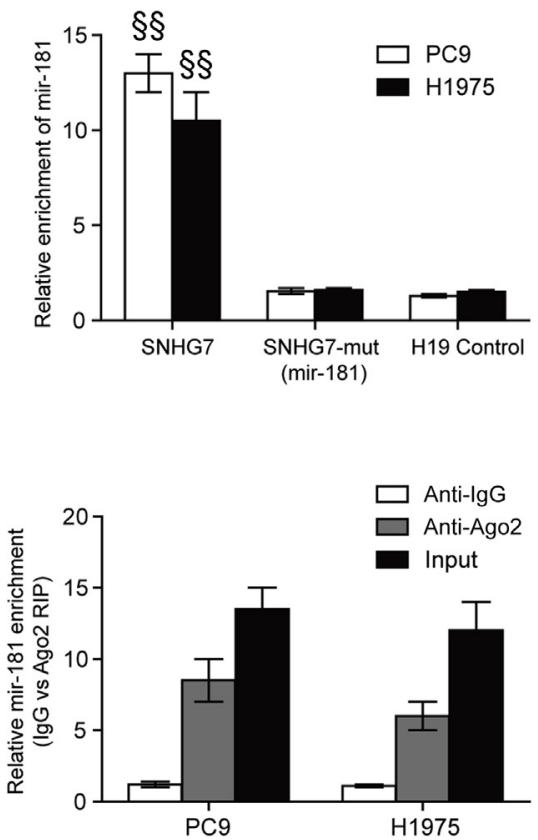

C

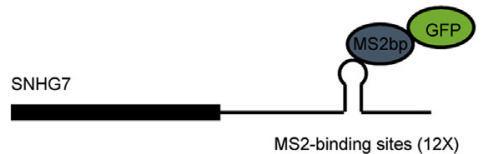

SNHG7-mut (mir-181)

608-627
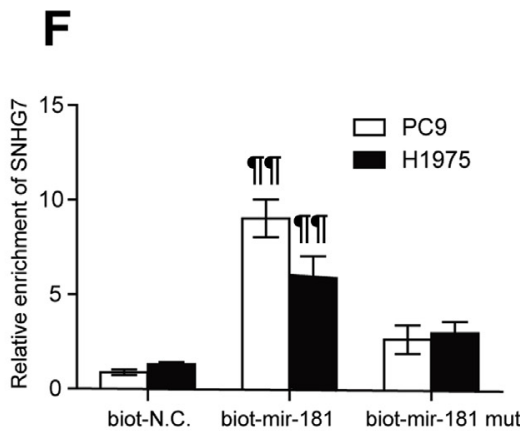

H

$\mathbf{F}$

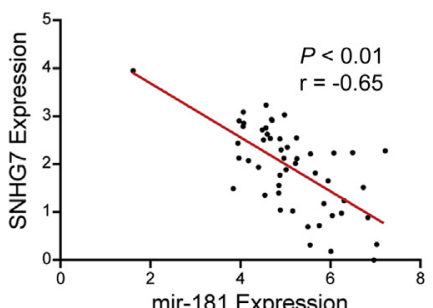

Figure 2 Small nucleolar RNA host gene 7 (SNHG7) is a direct target of microRNA mir-181. A: Relative luciferase activity of wild-type (WT) and mutant (mut) SNHG7 3'-untranslated region (UTR). The vertical lines in the top panel indicate the interaction between bases. B: mir-181 expression after SNHG7 knockdown and overexpression. C: Construct of SNHG7 transcripts combined with MS2 binding site. D: mir-181 enrichment was detected by MS2-based RNA immunoprecipitation (RIP). E: mir-181 enrichment was detected by RNA pulldown through biotin-labeled SNHG7 probe. F: SNHG7 enrichment was detected by RNA pulldown through biotin-labeled mir-181. G: SNHG7 and mir-181 enrichment was detected by argonaute 2 (Ago2)-based RIP. H: Correlation between SNHG7 and mir-181 in clinical samples. Red line indicates the regression line. ${ }^{* *} P<0.01$ versus mimics-NC; ${ }^{\dagger \dagger} P<0.01$; ${ }^{\ddagger \ddagger} P<0.01$ versus MS2-SNHG7-mut (mir-181); ${ }^{\S \S} P<0.01$ versus SNHG7-mut (mir-181); ${ }^{\boldsymbol{\top} \uparrow} P<0.01$ versus biot-N.C. Ctl, control; KD, knockdown.

prediction, luciferase assays were performed. Only wildtype SNHG7 could bind mir-181, but not SNHG7 with mutations in its MRE (Figure 2A). SNHG7 knockdown increased cellular mir-181 expression, whereas SNHG7 overexpression down-regulated mir-181 level (Figure 2B). To validate the direct binding between SNHG7 and mir181, SNHG7 transcripts combined with MS2-binding sites were constructed (Figure 2C). This construct was cotransfected into LUAD cells with MS2bp-GFP plasmid, and then GFP antibody was used for immunoprecipitation. SNHG7 significantly enriched mir-181 (Figure 2D). The interaction between SNHG7 and mir-181 was further validated by biotin-labeled SNHG7 probe (Figure 2E). Biotin-labeled mir-181 was used in inverse pulldown to explore whether mir-181 could pull down SNHG7. Only wild-type mir-181 could pull down SNHG7 (Figure 2F). Ago2 was reported to act as a bridge between lncRNA and miRNA. ${ }^{16}$ Ago2-RIP was used to test the binding between IncRNA and miRNA. ${ }^{17}$ In this study also, the Ago2-RIP assay was used. SNHG7 and mir-181 were enriched by Ago2-RIP as compared with IgG (Figure 2G). Lastly, a negative correlation was found between SNHG7 and mir-181 in LUAD 
samples (Figure 2H). These results indicated the direct binding between SNHG7 and mir-181.

\section{SNHG7 Inhibits Cell Proliferation and Migration through Competitively Binding to mir-181}

Gene ontology analysis was performed on differently expressed genes between SNHG7-high and SNHG7-low groups in LUAD samples from the TCGA. Cell proliferation and adhesion were involved in these differently expressed genes (Figure 3A). Colony formation and transwell assays were then performed. SNHG7 knockdown increased cell proliferation and cell migration, and this trend was reversed by mir-181 knockdown (Figure 3, B and C). mir-181 could rescue the suppression of cell proliferation and cell migration induced by SNHG7 overexpression (Figure 3, B and C). As the epithelial-mesenchymal transition (EMT) process and $\mathrm{Wnt} / \beta$-catenin pathway was activated in various kinds of tumors, cell proliferation and migration were promoted. Thus, this study investigated the regulation of SNHG7 on the EMT and the Wnt//-catenin pathway. SNHG7 knockdown increased the EMT process and activated Wnt/ $\beta$-catenin downstream genes (Figure 3D). SNHG7 overexpression showed the opposite effect on EMT and Wnt/ $\beta$-catenin pathway. These results indicated that SNHG7 inhibits cell proliferation and migration through competitively binding to mir- 181 .

\section{mir-181 Directly Targets cbx7}

Based on predictions from MiRanda, TargetScan, and PicTar, cbx7 was selected on the basis of its involvement in carcinogenesis in LUAD. To validate that mir-181 directly targets cbx7, dual-luciferase assays were performed. The luciferase activity of the wild-type cbx7 $3^{\prime}$-UTR was significantly decreased by mir-181, whereas the activity of the mutant cbx7 3 '-UTR was not changed by mir-181 (Figure 4A). Additionally, mir-181 can complement the seed region of cbx7, and the MRE of SNHG7 was found to be the same as the $\operatorname{cbx} 7$ seed region (Figure $4 \mathrm{~B}$ ). To further validate the correlation of mir- 181 and $\operatorname{cbx} 7$, the expression of mir-181 and cbx7 in tumor tissues and adjacent nontumor tissues was detected. The expression of mir-181was negatively correlated with $\operatorname{cbx} 7$ (Figure $4 \mathrm{C}$ ). The results of the Western blots showed that mir-181 promoted the EMT process, whereas cbx7 reversed this trend (Figure 4D). Colony formation and migration assays also showed that mir-181 overexpression promoted cell proliferation and migration, but cbx7 showed the opposite effect (Figure 4, E and $\mathrm{F}$ ).

\section{Down-Regulation of $\mathrm{cbx7}$ Is Correlated with LUAD Progression}

LUAD sequencing data from the TCGA database was analyzed. cbx7 was down-regulated in LUAD compared with adjacent nontumor tissues (Figure 5A and Supplemental Figure S2). Kaplan-Meier analysis of TCGA database showed that low expression of $\mathrm{cbx} 7$ indicated poorer survival in LUAD patients (Figure 5B). Cbx7 expression was also validated in clinical samples using qPCR (Figure 5C). Colony formation and transwell assays were then performed. Compared with the control group, cbx7 knockdown increased cell proliferation and migration (Figure 5, D and E). In addition, cbx7 overexpression decreased colony numbers and cell migration.

\section{cbx7 Suppresses Wnt/ $\beta$-Catenin Pathway in LUAD}

The Wnt/ $\beta$-catenin pathway was activated in various kinds of tumors and promoted cell proliferation and migration. Therefore, the regulation of $\operatorname{cbx} 7$ on the $W n t / \beta$-catenin pathway was investigated. First, a canonical top-flash reporter system was used to detect the activity of the $\mathrm{Wnt} / \beta$ catenin pathway. The cbx7 knockdown activated the Wnt pathway, whereas cbx7 overexpression suppressed Wnt pathway activity (Figure 6A). Furthermore, direct downstream genes of the $\mathrm{Wnt} / \beta$-catenin pathway such as $c-m y c$, cyclin D1, c-jun, fra-1, and MMP-7 were also detected. These genes were activated by the cbx7 knockdown, and cbx7 overexpression suppressed the expression of these genes (Figure 6B). It was also found that cbx7 knockdown promoted EMT, whereas cbx7 overexpression inhibited EMT (Figure 6C). The authors then explored the mechanism of how cbx7 suppressed the Wnt/ $\beta$-catenin pathway. Kim et $a^{18}$ reported that $\operatorname{cbx} 7$ inhibited the $\mathrm{Wnt} / \beta$-catenin pathway through DKK-1. However, in this study, the authors observed a direct interaction between cbx7, TCF4, and $\beta$-catenin (Figure 6D), and cbx7 decreased the endogenous interaction between TCF4 and $\beta$-catenin (Figure 6E). Taken together, these results suggest that cbx 7 may inhibit $\mathrm{Wnt} / \beta$ catenin pathway by inhibiting the formation of TCF 4 and $\beta$ catenin complex in LUAD.

\section{IncRNA-SNHG7 Is Regulated by Methylation of Promoter}

To explore the mechanism that induced down-regulated SNHG7 in LUAD, the SNHG7 promoter was analyzed. The UCSC Genome Browser showed that $\mathrm{CpG}$ islands were enriched in the SNHG7 promoter (Figure 7A). Methylation data from TCGA also showed a hypermethylation status in the SNHG7 promoter in tumor samples as compared with normal samples (Figure 7A). Furthermore, bisulfate sequencing showed that compared with SNHG7 highexpression samples, SNHG7 low-expression samples had more methylated $\mathrm{CpG}$ sites in their promoters (Figure 7B). In cell lines, 5-aza, a reagent that inhibits DNA methyltransferases (DNMTs) and induces hypomethylation in gene promoters, was used. 5-aza clearly reduced DNA methylation in the SNHG7 promoter (Figure $7 \mathrm{C}$ ). These results 
A
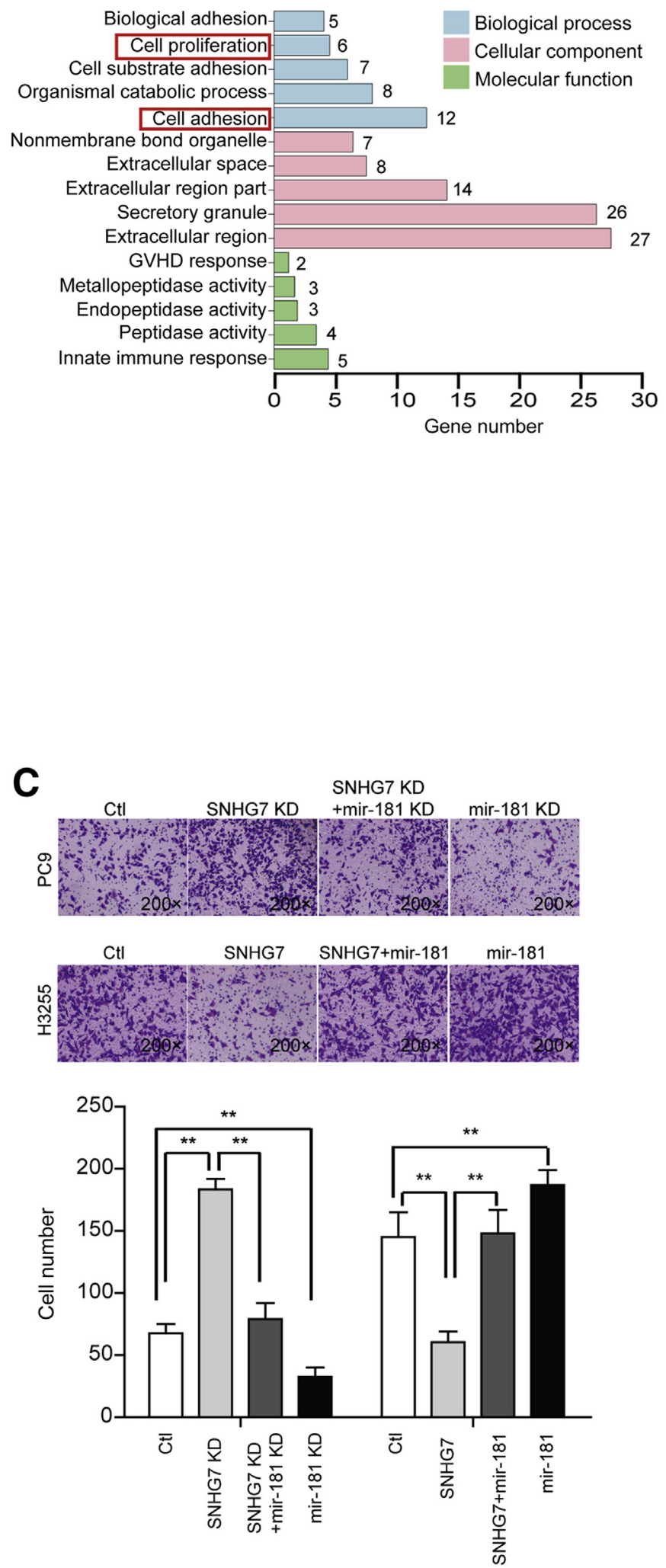

B
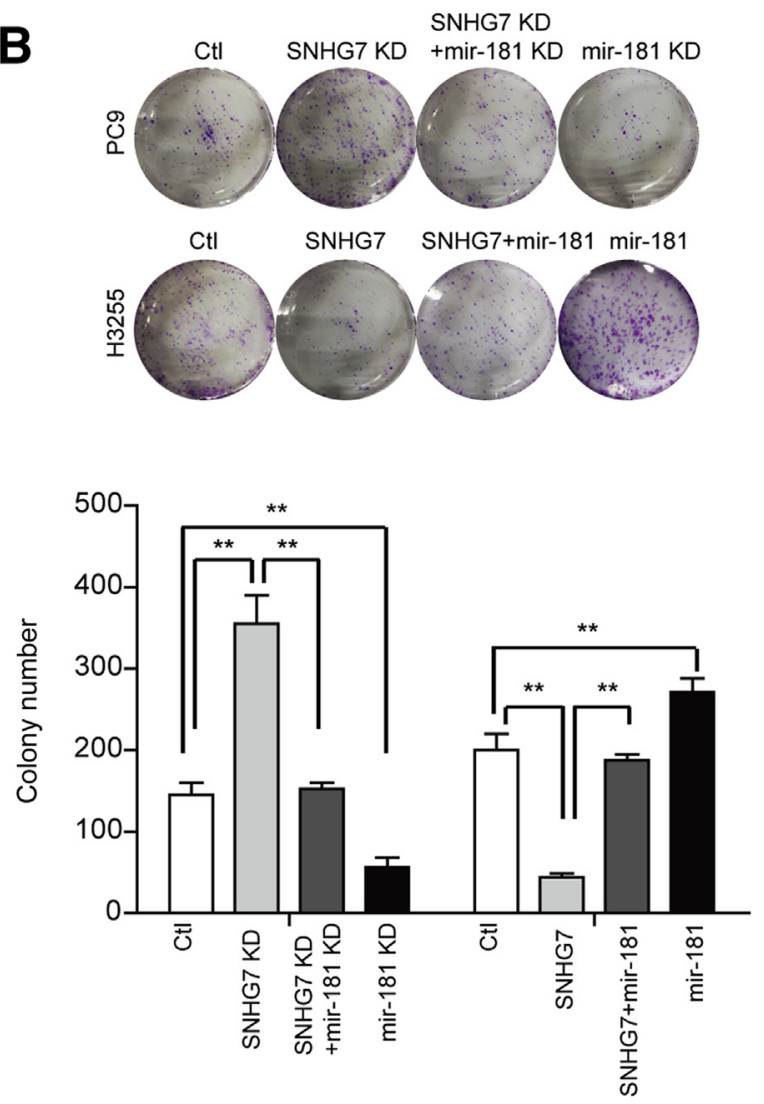

D

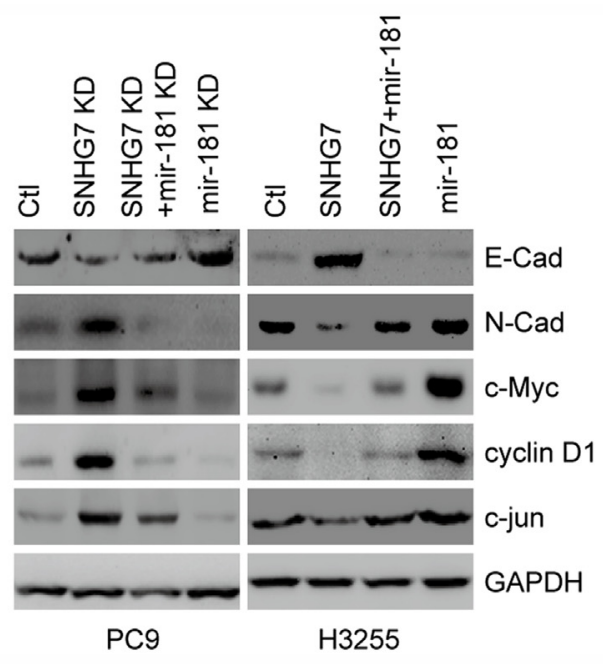

Figure 3 Small nucleolar RNA host gene 7 (SNHG7) inhibits cell proliferation and migration through competitively binding to microRNA mir-181. A: Gene ontology $\mathrm{GO}$ analysis of genes between SNHG7 high group and low group. Red boxes indicate the target biological process. B: Colony formation in cells with SNHG7 knockdown (KD) or overexpression. C: Cell migration in cells with SNHG7 knockdown or overexpression. D: Alteration of epithelial-mesenchymal transition (EMT) markers and Wnt/ $\beta$-catenin down-stream genes in cells with SNHG7 knockdown and overexpression. ${ }^{* *} P<0.01$. Original magnification, $\times 200$ (C). Ctl, control. 
A

B

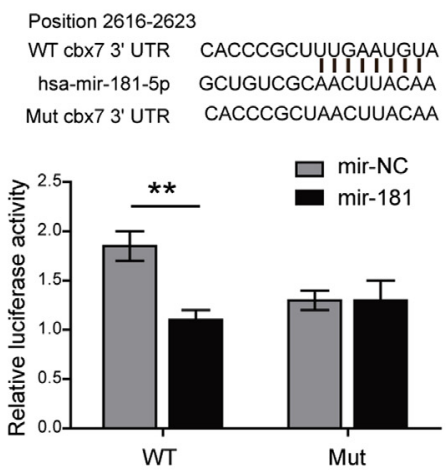

D

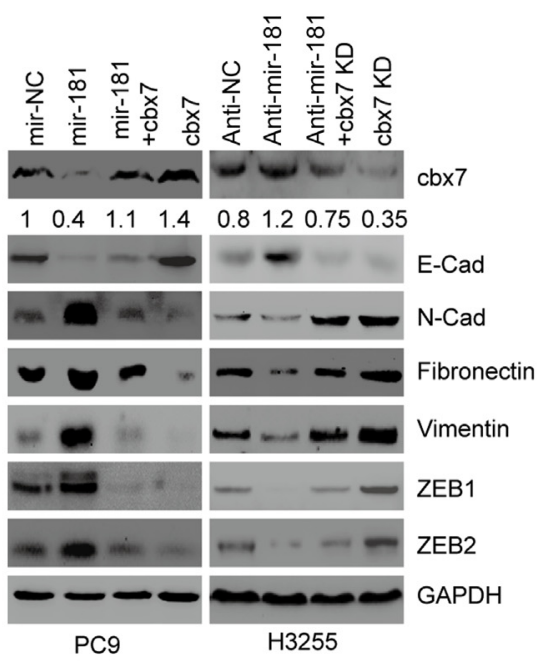

E
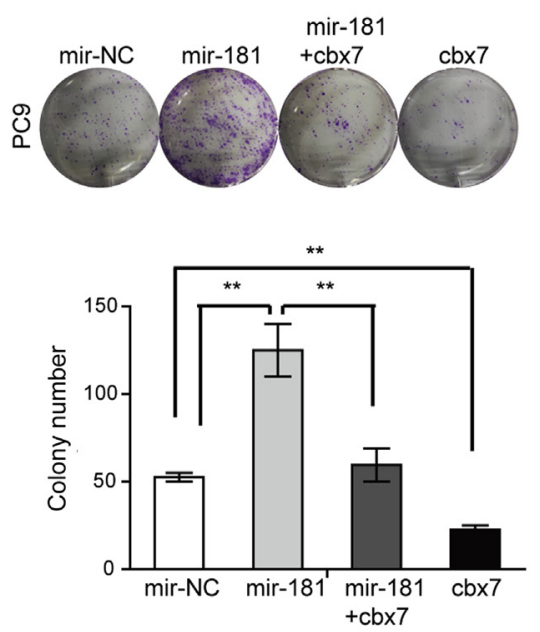

WT SNHG7: 5' cugCCCCGUGAACGUUUGAAUGU u 3' mir-181: 3' ugaGUGGCUGUCGC--AACUUACAa 5'

WT cbx73' UTR CACCCGCUU UGAAUGUA hsa-mir-181-5p GCUGUCGCAACUUACAA
C

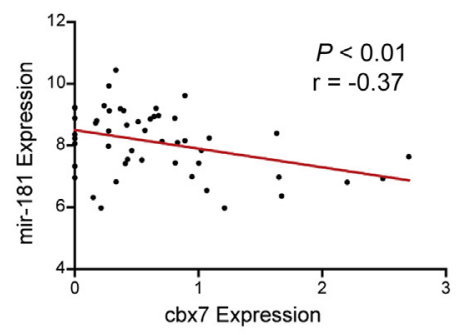

Anti-mir-181

$$
+
$$
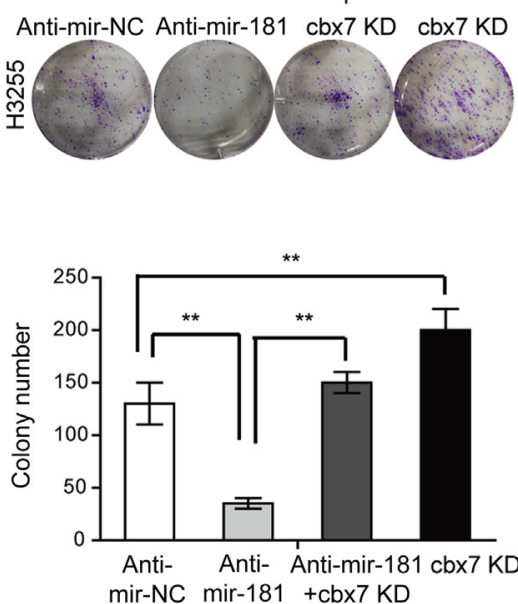

F
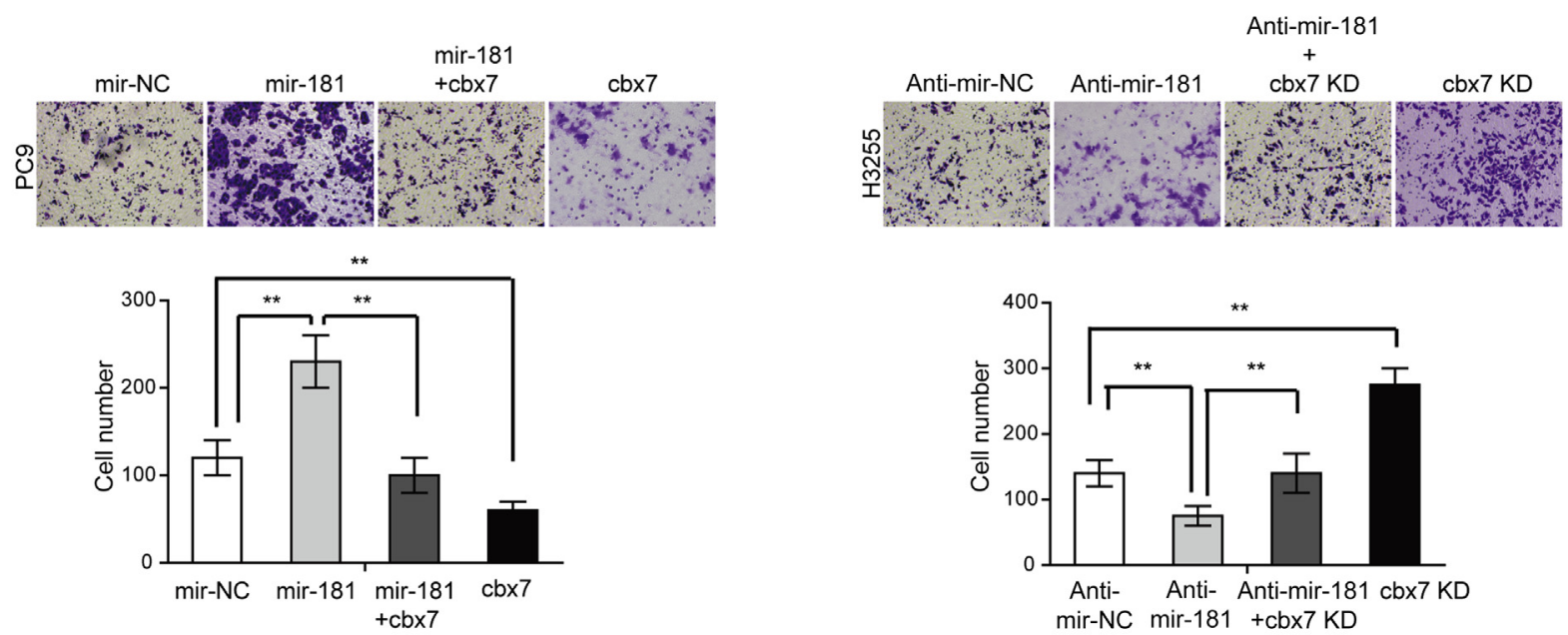

Figure 4 A: Relative luciferase activity of wild-type (WT) and mutant (mut) cbx7 $3^{\prime}$-untranslated region (UTR). Vertical lines indicate the interaction between bases. B: mir-181 can complement the seed region of $\mathrm{cbx} 7$, which is similar to the small nucleolar RNA host gene 7 (SNHG7) miRNA response element (MRE). Red boxes indicate the seed region of cbx7 and the MRE of SNHG7; vertical lines, the interaction between bases. C: Correlation between SNHG7 and mir-181 expression. Red line indicates the regression line. D: Alteration of epithelial-mesenchymal transition (EMT) markers in cells with mir-181 knockdown and overexpression. E and F: Colony formation and cell migration in cells with mir-181 knockdown (KD) or overexpression. ${ }^{* *} P<0.01$. Original magnification, $\times 200(\mathbf{F})$. 
A

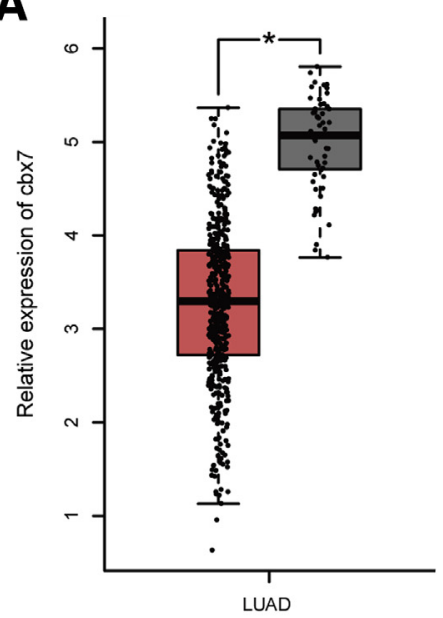

B

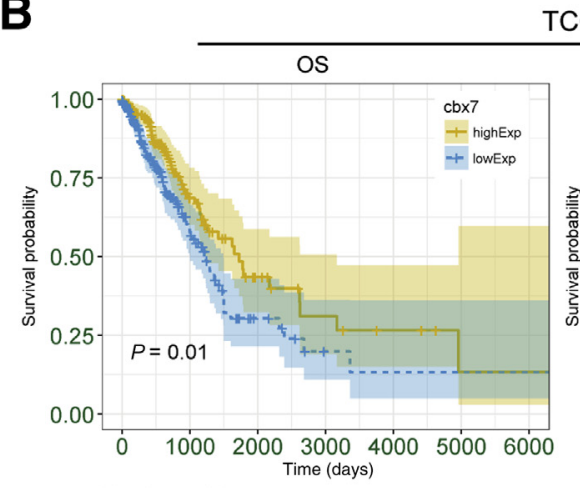

TCGA
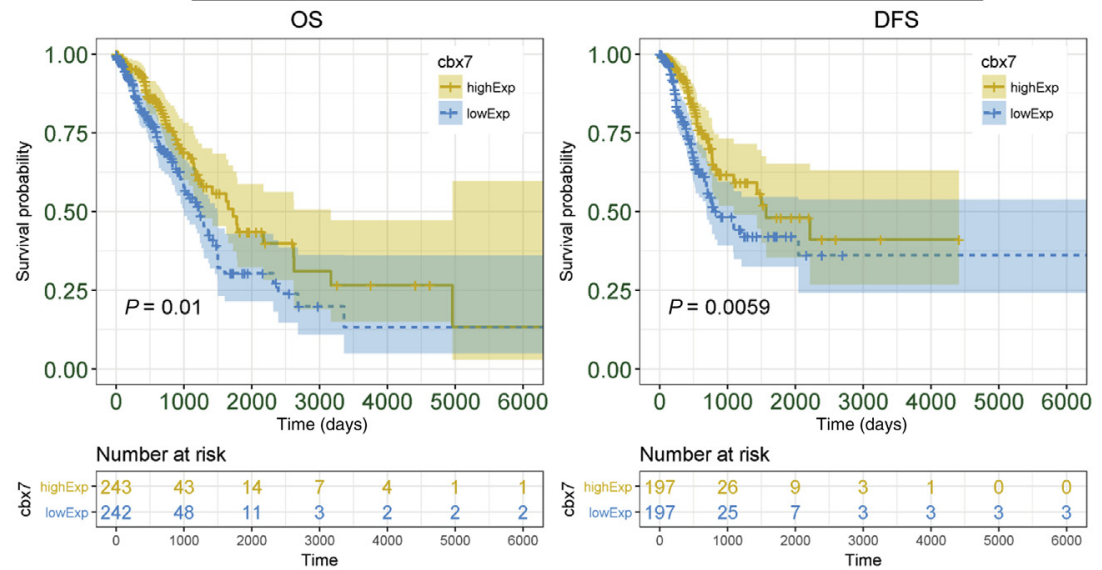

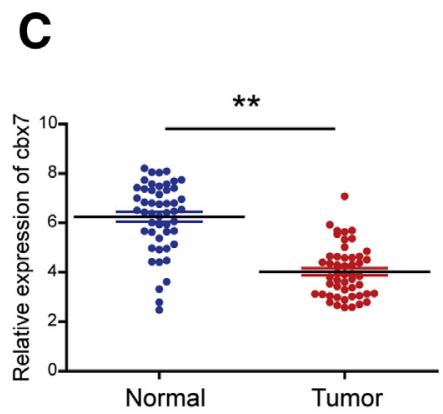

D
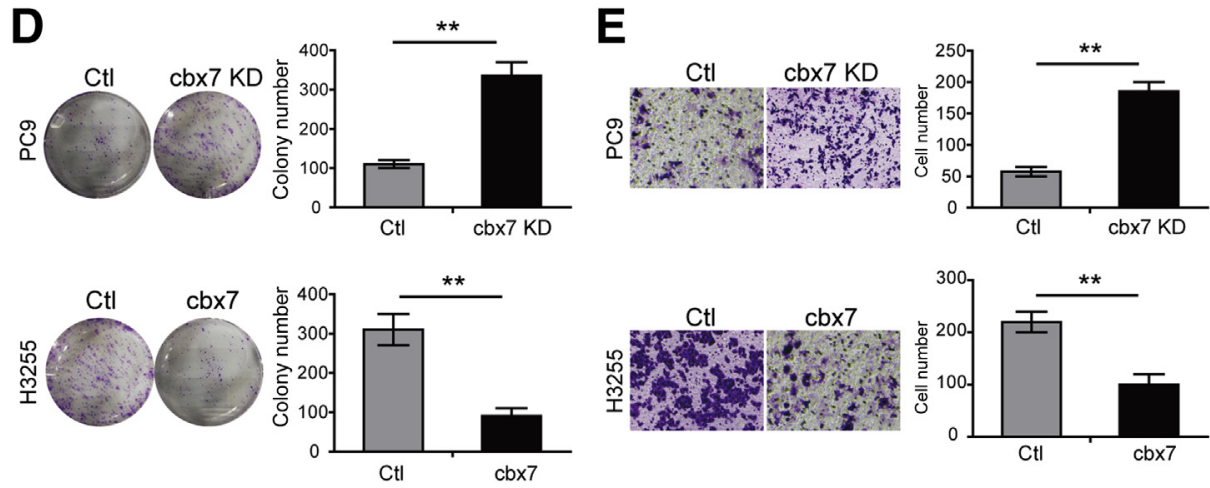

Figure 5 Down-regulation of $c b \times 7$ is correlated with lung adenocarcinoma (LUAD) progression. $A: c b x 7$ is down-regulated in LUAD in the Cancer Genome Atlas (TCGA) database. B: Kaplan-Meier curve of LUAD patients with cbx7 high expression (highExp) and low expression (lowExp). C: Small nucleolar RNA host gene 7 (SNHG7) expression in clinical samples. Black lines indicate the mean expression (A and C). D and E: Colony formation and cell migration in cells with cbx7 knockdown (KD) or overexpression. $n=483$ tumor $(\mathbf{A}) ; n=59$ normal $(\mathbf{A})$. ${ }^{*} P<0.05,{ }^{*} P<0.01$. Original magnification, $\times 200(E)$. Ctl, control; DFS, disease free survival; OS, overall survival.

indicate that SNHG7 expression may be regulated by $\mathrm{CpG}$ methylation in the promoter.

A xenograft mouse model was used to explore the effects of SNHG7 on tumor formation in vivo. Compared with the control group, SNHG7 knockdown and mir-181 overexpression significantly increased tumor volume and tumor weight (Figure 7D). Relative mRNA expression of cbx7, E-cadherin, and N-cadherin in the harvested tumor tissues was analyzed by qPCR (Figure 7E).

\section{Discussion}

Accumulating attention has been attracted to IncRNA because of its crucial role in gene regulation and cell process. In the present study, the authors identified a set of differently expressed lncRNAs in LUAD as compared with adjacent nontumor tissues, using the TCGA and GTEx databases. The low expression of SNHG7, a down-regulated lncRNA, is correlated with poor prognosis in LUAD. SNHG7 expression was down-regulated in LUAD tissues and cell lines. The authors also detected a biological effect of SNHG7 in LUAD cell lines. SNHG7 knockdown, not only increased cell proliferation, but also promoted cell migration. SNHG7 overexpression showed the opposite effect in cell lines. In vivo, SNHG7 knockdown increased tumor volume and tumor weight.

The crosstalk between lncRNA and mRNA was mediated by competing MREs. Hu et $\mathrm{al}^{19}$ showed that the lncRNA HOTAIR promotes osteoarthritis progression through acting as a ceRNA. HOTAIR sponges mir-17 and increases the expression of alpha-1, 2 fucosyltransferase 2 (FUT2). In LUAD, IncRNA-UCA1 exerts oncogenic functions by targeting mir-193. ${ }^{20}$ In this study, bioinformatics analysis predicted that SNHG7 contains an mir181 binding site. The following luciferase assay, RIP, and RNA pulldown all demonstrated SNHG7 could significantly enrich mir-181. But SNHG7 with a mutation in MRE could not associate with mir-181. Cellular functional assay revealed that mir-181 overexpression markedly increased cell proliferation and migration through directly targeting $\operatorname{cbx} 7$, a critical tumor suppressor. In vivo, it was also found that SNHG7 acts as a tumor suppressor and mir-181 promotes tumorigenesis. 


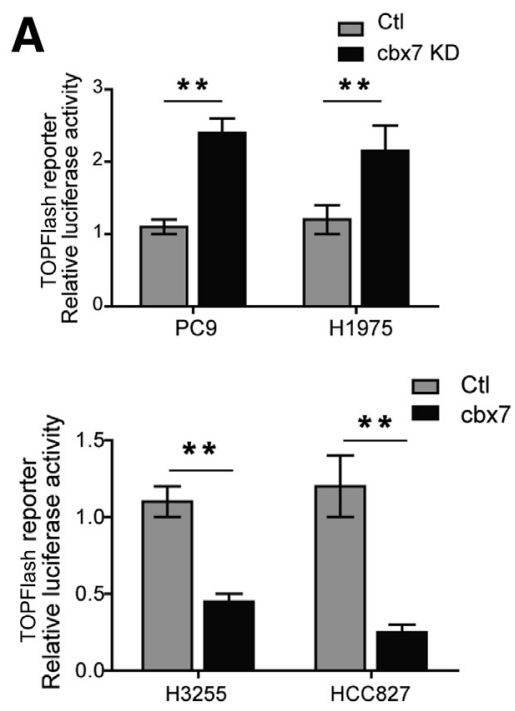

D
B
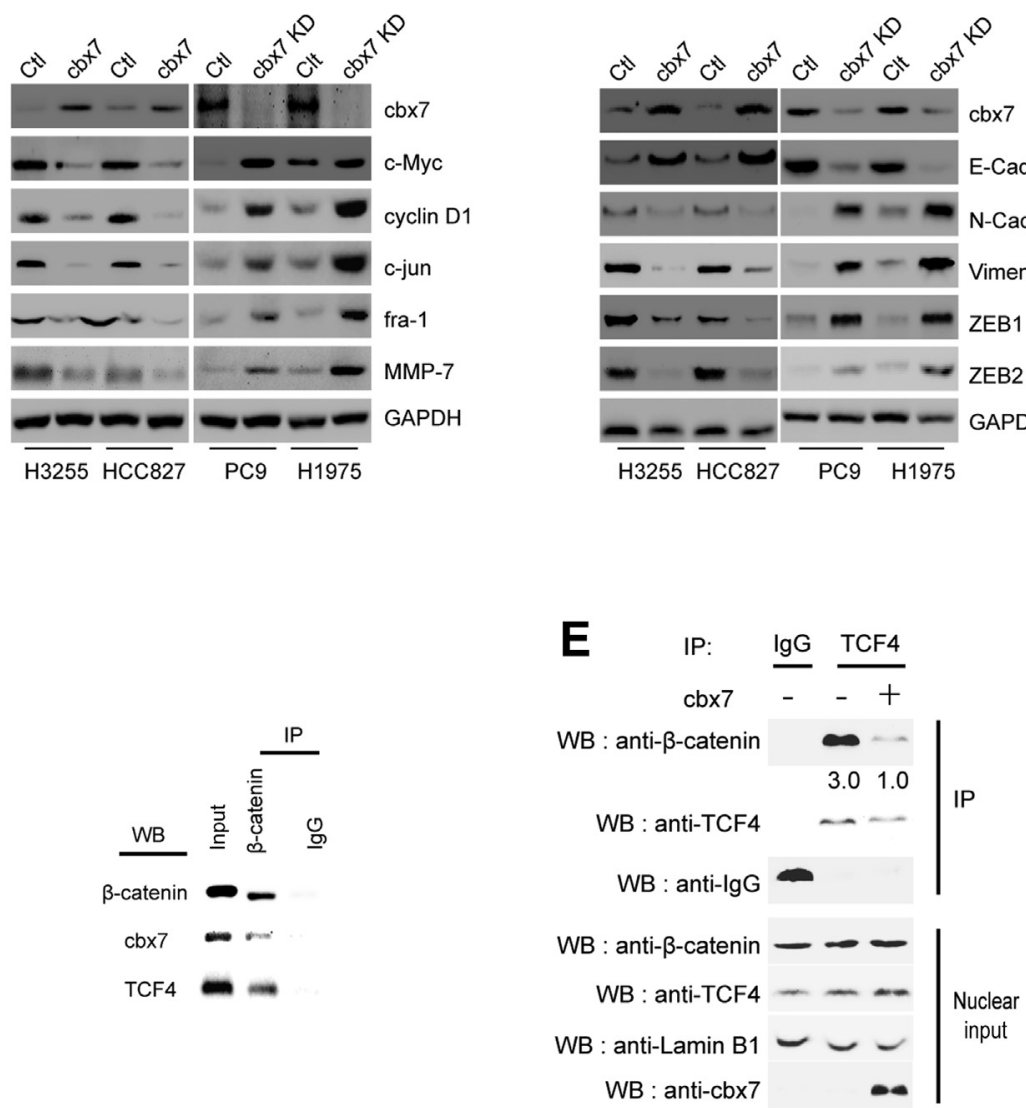

Figure 6 A: TOPFlash reporter assay in cells with cbx7 knockdown (KD) and overexpression. B: Alteration of Wnt/ $\beta$-catenin downstream genes in cells with cbx7 knockdown and overexpression. C: Alteration of epithelial-mesenchymal transition (EMT) markers in cells with cbx7 knockdown (KD) and overexpression. D and E: Coimmunoprecipitation of cbx7, transcription factor 4 (TCF4), and $\beta$-catenin. ${ }^{* *} P<0.01$. Ctl, control; IP, immunoprecipitation; WB, Western blot.

Finally, it was demonstrated that SNHG7 inhibits LUAD proliferation and metastasis in a mir-181-dependent way.

In previous studies, SNHG7 was reported to be an oncogene. $\mathrm{Li}$ et $\mathrm{al}^{11}$ reported that $\mathrm{SNHG7}$ promotes colorectal cancer progression. She et $\mathrm{al}^{21}$ reported that SNHG7 acted as a tumor activator. But in the current study, it was found that SNHG7 inhibited tumorigenesis in LUAD. First, SNHG7 exhibits cellular- and tissue-specific functions in a context-dependent manner, which may explain the contradiction between the antitumor function of SNHG7 observed in this study and its pro-tumor action in Li et al's study. ${ }^{11}$ Second, to expand the sample size, the TCGA and GTEx databases were used, which could supply more convincing data. Unlike She et al's study, ${ }^{21}$ the current study found that SNHG7 expression was downregulated in tumor tissues as compared with adjacent nontumor tissues. In the current study, SNHG7 plays a role as a tumor suppressor. These difference between the current study and She et al's report ${ }^{21}$ may be attributed to sample size. She et al's study used a small sample size ( $n=25$ tumor, $n=25$ normal), whereas the current study used the TCGA and GTEx databases $(n=483$ tumor, $n=347$ normal). Thus, SNHG7 may play different roles depending on the cancer type, and the antitumor effect of SNHG7 in this study was based on a large-sample investigation.

This research also has limitations. First, it was found that the lncRNA SNHG7 was regulated by the methylation status of its promoter. It has been reported that lncRNA could be regulated by transcriptional factors. ${ }^{22}$ In the UCSC Genome Browser, it was also found transcriptional factor binding sites in the SNHG7 promoter. Thus, whether SNHG7 could be regulated by transcriptional factors needs further investigation. Second, it was found that mir-181 was regulated by SNHG7. But, through bioinformatic analysis, it was found that mir-181 could be regulated by other lncRNAs such as SNHG5, MALAT1, and XIST. It is unknown whether these lncRNAs exert their functions through mir-181 in LUAD. 
A
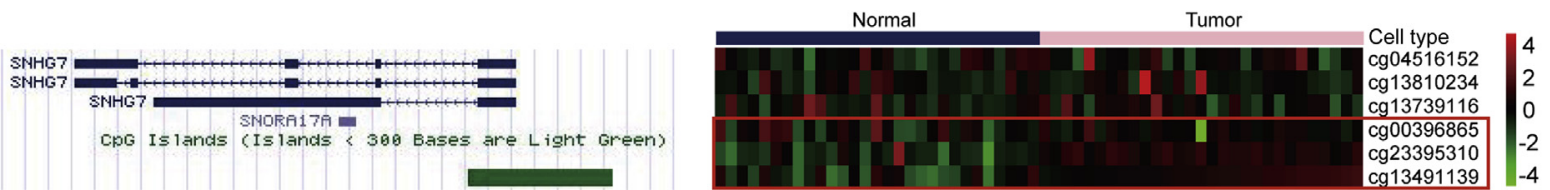

B
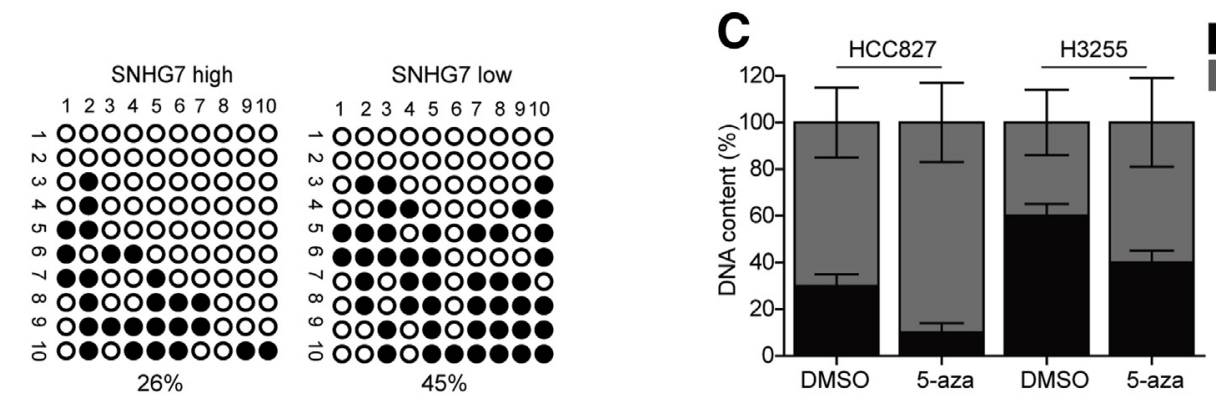

D
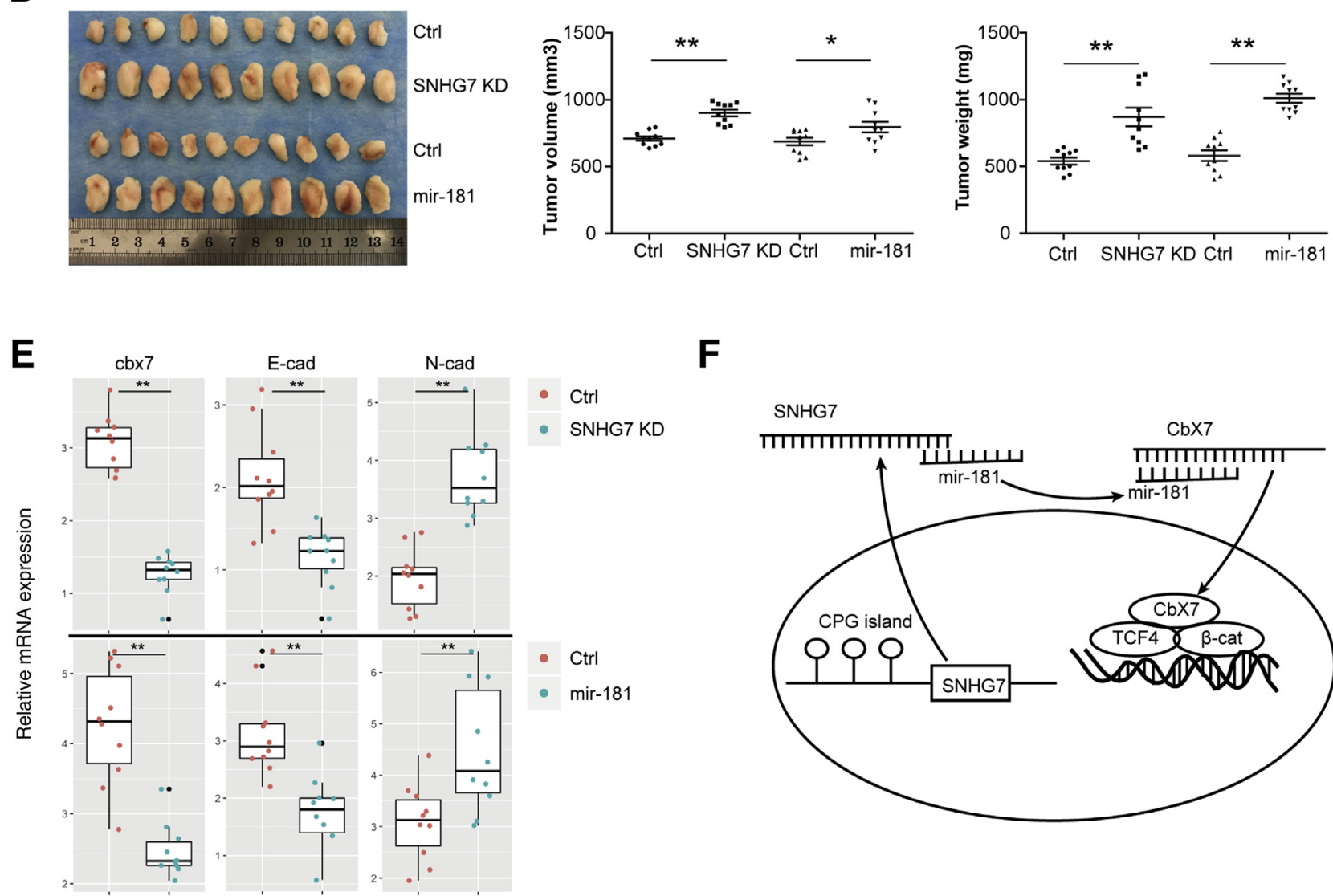

$\mathbf{F}$

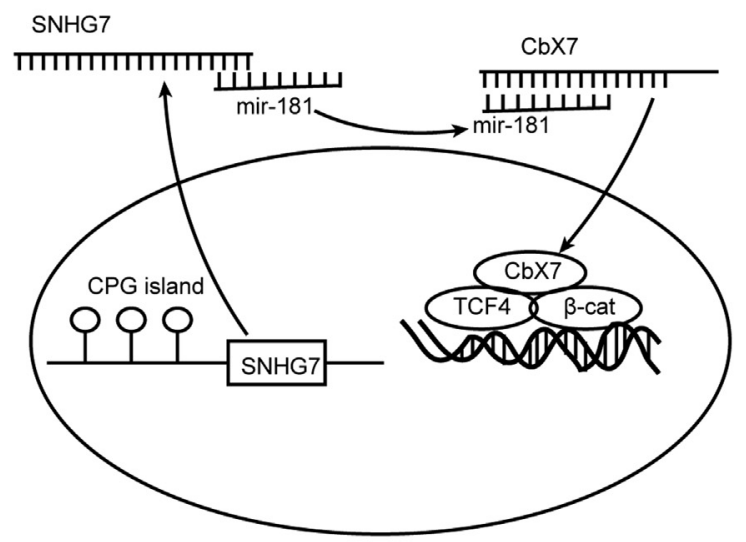

Figure 7 Long noncoding (lnc) RNA small nucleolar RNA host gene 7 (IncRNA-SNHG7) is regulated by methylation of the promoter. A: CpG islands of the SNHG7 promoter in the UCSC Genome Browser (left panel). DNA methylation analysis of SNHG7 in lung adenocarcinoma (LUAD) and corresponding normal tissues generated from the $450 \mathrm{k}$ DNA methylation data in the Cancer Genome Atlas (TCGA) database (right panel). Red box indicates the differently expressed methylation sites. B: Bisulfate sequencing of SNHG7 high-expression and low-expression samples. C: Methylated and unmethylated DNA content in the SNHG7 promoter of cell lines treated with 5-aza. D: Tumor volume and tumor weight in a xenograft mouse model of SNHG7 knockdown (KD) and microRNA mir-181 overexpression. E: Relative mRNA expression of cbx7, E-cadherin, and N-cadherin in the harvested tumor tissues. Black lines indicate the mean expression (D and E); black dots, outliers (D and E). F: Schematic presentation of crosstalk between long noncoding RNA (IncRNA)-SNHG7/miRNA-181/cbx7 in LUAD. ${ }^{*} P<0.05,{ }^{*} P<0.01$. Ctrl, control.

Collectively, in the present study, crosstalk between lncRNA-SNHG7/miRNA-181/cbx 7 was demonstrated. SNHG7 exerts its function as a ceRNA through interacting with mir-181. This process protected cbx7
mRNA from degradation by mir-181 (Figure 7F). The effectiveness and safety of the lncRNA-SNHG7/miRNA$181 / \mathrm{cbx} 7$ pathway in LUAD therapy needs further investigation. 


\section{Supplemental Data}

Supplemental material for this article can be found at http://doi.org/10.1016/j.ajpath.2020.02.011.

\section{References}

1. Peng Z, Wang J, Shan B, Li B, Peng W, Dong Y, Shi W, Zhao W, He D, Duan M, Cheng Y, Zhang C, Duan C: The long noncoding RNA LINC00312 induces lung adenocarcinoma migration and vasculogenic mimicry through directly binding YBX1. Mol Cancer 2018, 17:167

2. Jin X, Chen Y, Chen H, Fei S, Chen D, Cai X, Liu L, Lin B, Su H, Zhao L, Su M, Pan H, Shen L, Xie D, Xie C: Evaluation of tumorderived exosomal miRNA as potential diagnostic biomarkers for early-stage non-small cell lung cancer using next-generation sequencing. Clin Cancer Res 2017, 23:5311-5319

3. Forloni M, Gupta R, Nagarajan A, Sun L-S, Dong Y, Pirazzoli V, Toki M, Wurtz A, Melnick MA, Kobayashi S, Homer RJ, Rimm DL, Gettinger SJ, Politi K, Dogra SK, Wajapeyee N: Oncogenic EGFR represses the TET1 DNA demethylase to induce silencing of tumor suppressors in cancer cells. Cell Rep 2016, 16:457-471

4. Zhang H, Diab A, Fan H, Mani SKK, Hullinger R, Merle P, Andrisani O: PLK1 and HOTAIR accelerate proteasomal degradation of SUZ12 and ZNF198 during hepatitis B virus-induced liver carcinogenesis. Cancer Res 2015, 75:2363-2374

5. Tseng Y-Y, Moriarity BS, Gong W, Akiyama R, Tiwari A, Kawakami H, Ronning P, Reuland B, Guenther K, Beadnell TC, Essig J, Otto GM, O’Sullivan MG, Largaespada DA, Schwertfeger KL, Marahrens Y, Kawakami Y, Bagchi A: PVT1 dependence in cancer with MYC copy-number increase. Nature 2014, 512:82-86

6. Yang F, Bi J, Xue X, Zheng L, Zhi K, Hua J, Fang G: Up-regulated long non-coding RNA H19 contributes to proliferation of gastric cancer cells. FEBS J 2012, 279:3159-3165

7. Cesana M, Cacchiarelli D, Legnini I, Santini T, Sthandier O, Chinappi M, Tramontano A, Bozzoni I: A long noncoding RNA controls muscle differentiation by functioning as a competing endogenous RNA. Cell 2011, 147:358-369

8. Zhuang LK, Yang YT, Ma X, Han B, Wang ZS, Zhao QY, Wu LQ, Qu ZQ: MicroRNA-92b promotes hepatocellular carcinoma progression by targeting Smad7 and is mediated by long non-coding RNA XIST. Cell Death Dis 2016, 7:e2203

9. Ding J, Yeh C-R, Sun Y, Lin C, Chou J, Ou Z, Chang C, Qi J, Yeh S: Estrogen receptor beta promotes renal cell carcinoma progression via regulating LncRNA HOTAIR-miR-138/200c/204/217 associated CeRNA network. Oncogene 2018, 37:5037-5053
10. Shan Y, Ma J, Pan Y, Hu J, Liu B, Jia L: LncRNA SNHG7 sponges miR-216b to promote proliferation and liver metastasis of colorectal cancer through upregulating GALNT1. Cell Death Dis 2018, 9:722

11. Li Y, Zeng C, Hu J, Pan Y, Shan Y, Liu B, Jia L: Long non-coding RNA-SNHG7 acts as a target of miR-34a to increase GALNT7 level and regulate $\mathrm{PI} 3 \mathrm{~K} / \mathrm{Akt} / \mathrm{mTOR}$ pathway in colorectal cancer progression. J Hematol Oncol 2018, 11:89

12. Pei Y-F, Yin X-M, Liu X-Q: TOP2A induces malignant character of pancreatic cancer through activating beta-catenin signaling pathway. Biochim Biophys Acta Mol Basis Dis 2018, 1864:197-207

13. Davis T, Vaisvila R: High sensitivity 5-hydroxymethylcytosine detection in Balb/C brain tissue. J Vis Exp 2011

14. Pei Y-F, Lei Y, Liu X-Q: MiR-29a promotes cell proliferation and EMT in breast cancer by targeting ten eleven translocation 1. Biochim Biophys Acta 2016, 1862:2177-2185

15. Wang Y-G, Wang T, Shi M, Zhai B: Long noncoding RNA EPB41L4A-AS2 inhibits hepatocellular carcinoma development by sponging miR-301a-5p and targeting FOXL1. J Exp Clin Cancer Res 2019, 38:153

16. Zhao C-C, Jiao Y, Zhang Y-Y, Ning J, Zhang Y-R, Xu J, Wei W, Kang-Sheng G: Lnc SMAD5-AS1 as ceRNA inhibit proliferation of diffuse large B cell lymphoma via Wnt/beta-catenin pathway by sponging miR-135b-5p to elevate expression of APC. Cell Death Dis 2019, 10:252

17. Chen S, Chen J-Z, Zhang J-Q, Chen H-X, Qiu F-N, Yan M-L, Tian YF, Peng C-H, Shen B-Y, Chen Y-L, Wang Y-D: Silencing of long noncoding RNA LINC00958 prevents tumor initiation of pancreatic cancer by acting as a sponge of microRNA-330-5p to down-regulate PAX8. Cancer Lett 2019, 446:49-61

18. Kim H-Y, Park J-H, Won H-Y, Lee J-Y, Kong G: CBX7 inhibits breast tumorigenicity through DKK-1-mediated suppression of the Wnt/beta-catenin pathway. FASEB J 2015, 29:300-313

19. Hu J, Wang Z, Shan Y, Pan Y, Ma J, Jia L: Long non-coding RNA HOTAIR promotes osteoarthritis progression via miR-17-5p/FUT2/ $\beta$ catenin axis. Cell Death Dis 2018, 9:711

20. Nie W, Ge H-J, Yang X-Q, Sun X, Huang H, Tao X, Chen W-S, Li B: LncRNA-UCA1 exerts oncogenic functions in non-small cell lung cancer by targeting miR-193a-3p. Cancer Lett 2016, 371: 99-106

21. She K, Yan H, Huang J, Zhou H, He J: miR-193b availability is antagonized by LncRNA-SNHG7 for FAIM2-induced tumour progression in non-small cell lung cancer. Cell Prolif 2018, 51: e12406

22. Wang Z-Q, Cai Q, Hu L, He C-Y, Li J-F, Quan Z-W, Liu B-Y, Li C, Zhu Z-G: Long noncoding RNA UCA1 induced by SP1 promotes cell proliferation via recruiting EZH2 and activating AKT pathway in gastric cancer. Cell Death Dis 2017, 8:e2839 